\title{
Development of a novel embryonic germline gene-related prognostic model of lung adenocarcinoma
}

\author{
Linjun Liu ${ }^{1}$, Ke Xu ${ }^{\text {Corresp., } 2}$, Yubai Zhou ${ }^{\text {Corresp. } 1}$ \\ ${ }^{1}$ Department of Biotechnology, College of Life Science \& Chemistry, Beijing University of Technology, Chaoyang, China, Beijing, China \\ 2 National Institute for Viral Disease Control and Prevention, China CDC, NHC Key Laboratory of biosafety, Beijing, China \\ Corresponding Authors: Ke Xu, Yubai Zhou \\ Email address: xuke@ivdc.chinacdc.cn, zhouyubai@bjut.edu.cn
}

Background: Emerging evidence implicates the correlation of embryonic germline genes with the tumor progress and patient's outcome. However, the prognostic value of these genes in lung adenocarcinoma (LUAD) has not been fully studied. Here we systematically evaluated this issue, and constructed a novel signature and a nomogram associated with embryonic germline genes for predicting the outcomes of lung adenocarcinoma.

Methods: The LUAD cohorts retrieved from The Cancer Genome Atlas (TCGA) and Gene Expression Omnibus (GEO) database were used as training set and testing set, respectively. The embryonic germline genes were downloaded from the website https://venn.lodder.dev. Then, the differentially expressed embryonic germline genes (DEGGs) between the tumor and normal samples were identified by limma package. The functional enrichment and pathway analyses were also performed by clusterProfiler package. The prognostic model was constructed by the least absolute shrinkage and selection operator (LASSO)-Cox regression method. Survival and Receiver Operating Characteristic (ROC) analyses were performed to validate the model using training set and four testing GEO datasets. Finally, a prognostic nomogram based on the signature genes was constructed using multivariate regression method. Results: Among the identified 269 DEGGs, 249 were up-regulated and 20 were down-regulated. GO and KEGG analyses revealed that these DEGGs were mainly enriched in the process of cell proliferation and DNA damage repair. Then, 103 DEGGs with prognostic value were identified by univariate Cox regression and further filtered by LASSO method. The resulting sixteen DEGGs were included in step multivariate Cox regression and an eleven embryonic germline gene related signature (EGRS) was constructed. The model could robustly stratify the LUAD patients into high-risk and low-risk groups in both training and testing set, and low-risk patients had much better outcomes. The multi-ROC analysis also showed that the EGRS model had the best predictive efficacy compared with other common clinicopathological factors. The EGRS model also showed robust predictive ability in 4 independent external 
datasets, and the area under curve (AUC) was 0.726 (GSE30219), 0.764 (GSE50081), 0.657 (GSE37745) and 0.668 (GSE72094). More importantly, the expression level of some genes in ERGS has a significant correlation with the progression of LUAD clinicopathology, suggesting these genes might play an important role in the progression of LUAD. Finally, based on EGRS genes, we built and calibrated a nomogram for conveniently evaluating patients' outcomes. 


\section{Development of a novel embryonic germline gene-related prognostic model of lung} adenocarcinoma

3

4 Linjun $\mathrm{Liu}^{1}, \mathrm{Ke} \mathrm{Xu}^{2 *}$, Yubai Zhou ${ }^{1 *}$

5 1. Department of Biotechnology, College of Life Science \& Chemistry, Beijing University of 6 Technology, Chaoyang, Beijing, China

7 2. NHC Key Laboratory of biosafety, National Institute for Viral Disease Control and Prevention,

8 China CDC, Changping, Beijing, China

$9 *$ Corresponding to:

$10 \mathrm{Ke} \mathrm{Xu,} \mathrm{Ph.D.,} \mathrm{NHC} \mathrm{Key} \mathrm{Laboratory} \mathrm{of} \mathrm{biosafety,} \mathrm{National} \mathrm{Institute} \mathrm{for} \mathrm{Viral} \mathrm{Disease} \mathrm{Control} \mathrm{and}$

11 Prevention, China CDC, Changbai 155\#, District of Changping, Beijing, 102206, China.

12 E-mail: xuke@ivdc.chinacdc.cn

13 Yubai Zhou, Ph.D., Department of Biotechnology, College of Life Science \& Chemistry, Beijing

14 University of Technology, Pingleyuan 100\#, District of Chaoyang, Beijing 100124, China.

15 E-mail: zhouyubai@bjut.edu.cn 
Abstract

Background: Emerging evidence implicates the correlation of embryonic germline genes with the tumor progress and patient's outcome. However, the prognostic value of these genes in lung adenocarcinoma (LUAD) has not been fully studied. Here we systematically evaluated this issue, and constructed a novel signature and a nomogram associated with embryonic germline genes for predicting the outcomes of lung adenocarcinoma. Methods: The LUAD cohorts retrieved from The Cancer Genome Atlas (TCGA) and Gene Expression Omnibus (GEO) database were used as training set and testing set, respectively. The embryonic germline genes were downloaded from the website https://venn.lodder.dev. Then, the differentially expressed embryonic germline genes (DEGGs) between the tumor and normal samples were identified by limma package. The functional enrichment and pathway analyses were also performed by clusterProfiler package. The prognostic model was constructed by the least absolute shrinkage and selection operator (LASSO)Cox regression method. Survival and Receiver Operating Characteristic (ROC) analyses were performed to validate the model using training set and four testing GEO datasets. Finally, a prognostic nomogram based on the signature genes was constructed using multivariate regression method. Results: Among the identified 269 DEGGs, 249 were up-regulated and 20 were downregulated. GO and KEGG analyses revealed that these DEGGs were mainly enriched in the process of cell proliferation and DNA damage repair. Then, 103 DEGGs with prognostic value were identified by univariate Cox regression and further filtered by LASSO method. The resulting sixteen DEGGs were included in step multivariate Cox regression and an eleven embryonic germline gene related signature (EGRS) was constructed. The model could robustly stratify the LUAD patients into high-risk and low-risk groups in both training and testing set, and low-risk patients had much better outcomes. The multi-ROC analysis also showed that the EGRS model had the best predictive efficacy compared with other common clinicopathological factors. The EGRS model also showed robust predictive ability in 4 independent external datasets, and the area under curve (AUC) was 0.726 (GSE30219), 0.764 (GSE50081), 0.657 (GSE37745) and 0.668 (GSE72094). More importantly, the expression level of some genes in ERGS has a significant correlation with the progression of LUAD clinicopathology, suggesting these genes might play an important role in the progression of LUAD. Finally, based on EGRS genes, we built and calibrated a nomogram for conveniently evaluating patients' outcomes.

\section{Introduction}

As the most common pathological subtype of non-small cell lung cancer (NSCLC), lung adenocarcinoma (LUAD) largely occurs among women and non-smoking populations(Herbst et al. 2018). Benefiting from the advance in surgical technique, targeted therapy, immunotherapy and radiochemotherapy, the treatment of LUAD has undergone revolutionary changes in recent years(Yang et al. 2020). However, due to the lack of sensitive early detection methods, most patients are in advanced stages at the time of their first diagnosis. The overall 5-year survival rate has not improved significantly(Chen et al. 2014). 
Many cancers activate genes normally associated with different developmental states, including germ cell-specific genes, which suggests that some unknown mechanisms which are normally limited to the development of germ cells maybe involved in the process of tumorigenesis(Bruggeman et al. 2018; Erenpreisa \& Cragg 2010; Kho et al. 2004; Lepourcelet et al. 2005; McFarlane \& Wakeman 2017; Simpson et al. 2005). With the first discovery of MAGE-1 in melanoma cells, a series of genes that are normally restricted to the testis, such as BAGE, GAGE, HOM-MEL-40, SYCP1, and NY-ESO-1 have been found in various tumors, and these genes are collectively called cancer/testis (CT) genes (Almeida et al. 2009; Boël et al. 1995; Chen et al. 1997; De Backer et al. 1999; Türeci et al. 1996; Türeci et al. 1998; van der Bruggen et al. 1991). Although the driving role of these CT genes in tumorigenesis and development has not been fully studied, there is evidence that they may play a role in the early stage of oncogenesis and the maintenance of tumor phenotypes(Bruggeman et al. 2018). However, these previously identified CT genes are mixed with genes derived from testicular somatic cells and are not truly germlinespecific genes. Currently, using bioinformatic methods, Bruggeman et.al. identified 672 so called embryonic germ cell genes which are restricted to the germline and also expressed in a variety of tumor tissues, including lung cancer(Bruggeman et al. 2020). However, the level of expression of these genes in lung adenocarcinoma and its relationship with patient prognostics have not been fully studied.

In this study, we systematically analyzed the expression levels of embryonic germline cellspecific genes in LUAD cohort and constructed a novel prognostic signature and nomogram, which can be used to predict the overall survival of LUAD patients.

\section{Material and Methods}

\section{Data source and preprocessing}

Data were collected and preprocessed as previously described(Yue et al. 2019). Briefly, as the training dataset, the expression profile and clinical information of LUAD cohort were downloaded from TCGA database using GDC tool and summarized into an expression and a clinical matrix, respectively. The ensemble ids in the expression matrix were converted into gene symbols according to the annotation file and the multiple expression data of a single gene were replaced by an average value. Genes that are not expressed in all samples are also removed from the expression matrix. For testing sets, we used lung adenocarcinoma as a keyword to search in the GEO database. After removing the datasets which are devoid of clinical data or expression data of genes in the model, four independent GEO datasets GSE30219, GSE50081, GSE37745 and GSE72094 were selected and downloaded via GEOquery package(Davis \& Meltzer 2007). For pan-cancer analyses, the expression profiles and clinical data of 33 TCGA cancer types were downloaded from TCGA database, and preprocessed according to previously mentioned procedures. Tumor cohorts with normal samples $<5$ were not included in the pan-cancer expression analysis. The gene expression values were all converted to transcripts per million transcripts (TPM) to facilitate subsequent analysis. The protein expression data of LUAD were obtained from the Clinical Proteomic Tumor Analysis Consortium (CPTAC) database (https://cptac-data-portal.georgetown.edu/). 
109 Identification of the differentially expressed embryonic germline genes (DEGGs)

110 The embryonic germline genes were downloaded from the website $111 \mathrm{https}$ //venn.lodder.dev(Bruggeman et al. 2020), and their expression data were extracted into a 112 matrix. Then, the differentially expressed embryonic germline genes (DEGGs) between tumor and 113 normal samples were identified using methods mentioned previously (Yue et al. 2019). The 114 multiple comparisons were controlled using the false discovery rate (FDR) (Benjamini \& 115 Hochberg 1995). The screening criteria were $\mid \log 2$ Fold Chang $\mid>1$ and $\mathrm{FDR}<0.05$.

\section{Functional enrichment and pathway analysis}

117 The Gene Ontology(GO) enrichment and Kyoto Encyclopedia of Genes and Genomes(KEGG) 118 pathway analysis were performed according to previously described methods (Yu et al. 2012; Yue 119 et al. 2019). P. adjust (FDR) $<0.05$ was considered statistically significant.

\section{Construction of EGRS in training dataset}

The EGRS model was established using methods described previously(Yue et al. 2019). Briefly, the 454 LUAD samples were included in the univariate Cox regression analysis to determine the prognostic value of DEGGs. $\mathrm{P}<0.05$ was considered statistically significant. The prognostic DEGGs were further screened out using LASSO regression via glmnet package in R software for avoiding model overfitting(Friedman et al. 2010). Then, the resulting key DEGGs were included in multivariate Cox analysis, and risk score formula was constructed as follow:

The $\beta$ and gene represent the coefficient of multivariate Cox regression and the expression value of corresponding DEGGs in EGRS, respectively. The * represents the multiplication operator.

\section{Validation of the EGRS in the training and testing sets}

To further validate the expression pattern, the protein expression profiles of DEGGs in EGRS between normal and tumor samples were analyzed using proteomic data of LUAD cohort obtained from CPTAC database. Then, the predicted effects of the EGRS was evaluated in the training and testing sets, respectively. Samples in the training set were divided into high- and low-risk group according to the median of risk score values. The risk score distribution, survival status and the expression level of 11 DEGGs in EGRS between the high- and low-risk samples were evaluated. In addition, we divided the patients in training set into two groups according to commonly used clinicopathological factors and analyze the differentiation of the expression levels of 11 DEGGs between the two groups by student's t-test. $\mathrm{P}<0.05$ was considered statistically significant. KaplanMeier (KM) survival analysis and receiver operating characteristic (ROC) curve were used to evaluate the predicting power of the EGRS, and the prognostic performance of other clinicopathological factors was also compared with that of EGRS model. The KM plot and ROC curve were also used to assess the generalization power of EGRS model in four independent GEO datasets. Finally, a pan-cancer expression and prognostic analyses of 11 DEGGs were performed to extend our findings to other types of cancers. 


\section{Construction and evaluation of a nomogram}

For predicting the OS of individual LUAD patient, Nomogram was generated based on the results of the multivariate Cox analysis to predict 1-, 3-, and 5-year OS. The performance of the nomogram was evaluated using calibration plots.

\section{Result}

\section{Identification the DEGGs in LUAD}

The transcriptome data of LUAD cohort which contains 497 tumor and 54 normal samples were downloaded from TCGA database, and the corresponding expression profiles of embryonic germline genes were extracted according to the data published previously(Bruggeman et al. 2020). The DEGGs between normal and tumor samples were identified by limma package in $\mathrm{R}$ software(Ritchie et al. 2015). Totally, 269 DEGGs were screened out, among which, the upregulated DEGGs were 249, and the down-regulated DEGGs were 20 (Fig. 1A, Supplementary TableS1). The representative DEGGs are shown by heat map (Figure 1B) and box plot (Figure $1 \mathrm{C})$, respectively.

\section{Functional enrichment and KEGG analysis of DEGGs}

The GO enrichment analysis of the DEGGs revealed that the DEGGs were mainly enriched in biological process of cell proliferation and DNA damage repair, such as nuclear division (GO:0000280), DNA replication (GO:0006260), cell cycle checkpoint (GO:0000075), doublestrand break repair (GO:0006302) and interstrand cross-link repair (GO:0036297) (Fig. 2A, B). The KEGG analysis showed that the enriched pathways were cell cycle (hsa04110), homologous recombination (hsa03440), and Fanconi anemia pathway (hsa03460) (Fig. 2C, D). The detailed GO terms of the three categories, biological process (BP), cellular component (CC), molecular function (MF) and KEGG results were presented in Supplementary TableS2 and Supplementary TableS3, respectively.

\section{The construction of embryonic germline gene related prognostic model}

For EGRS construction, the univariate Cox regression analysis was conducted and the resulting 103 DEGGs with prognostic value ( $\mathrm{p}<0.05$, Fig. $3 \mathrm{~A})$ were further screened by LASSO regression. Then, the sixteen key DEGGs were selected to performed the multivariate Cox regression analysis (Fig.3B, C). Finally, a prognostic signature containing eleven DEGGs (RAD54L, ZNF322, CENPI, IGF2BP1, IGF2BP3, RAD51AP1, E2F7, HMMR, DNAJC5B, ADAMTS12, NANOS1) was constructed and the risk score formula was presented as follow: Risk score $=(-0.4294) *$ RAD54L $-0.2411 *$ ZNF322 - $0.4645 *$ CENPI $+0.2243 *$ IGF2BP1 + $0.2095 * \mathrm{IGF} 2 \mathrm{BP} 3-0.5093 * \mathrm{RAD} 51 \mathrm{AP} 1+0.7979 * \mathrm{E} 2 \mathrm{~F} 7+0.7968 * \mathrm{HMMR}-0.3272 *$ DNAJC5B $+0.1997 *$ ADAMTS12 $-0.5248 *$ NANOS 1.

\section{The validation of the EGRS for survival prediction}


181 Due to the potential inconsistencies in gene expression between the transcriptome and the 182 proteome, we first verified the protein expression of the 11 DEGGs in EGRS in normal and tumor 183 samples through the CPTAC database. Given the limitations of available data, only 4 genes of the 11 DEGGs were analyzed. Consistent with the transcriptome data, the protein expression of 4 genes (IGF2BP1, IGF2BP3, HMMR and ADAMTS12) were up-regulated in LUAD tumor samples. The log2FoldChanges of IGF2BP1, IGF2BP3, HMMR and ADAMTS12 were 0.49, 1.14, 0.50 and 0.87 and the p-values of the 4 genes were 2.7E-02, 9.22E-08, 1.32E-07 and 7.27E-18, respectively. In addition, the pan-cancer expression analysis also revealed that 11 DEGGs in EGRS showed differential expression in most types of tumors, but diverse expression trends between tumor and paired adjacent normal tissues of some types of cancers were observed, suggesting that the DEGGs may play different roles in the development of different types of tumors (Supplementary figure S1). Then, Kaplan-Meier (KM) survival analysis indicated that the overall survival between the high- and low-risk groups in training set was significantly different, and the low-risk patients had significantly better outcomes than that of high-risk patients $(\mathrm{P}=4.083 \mathrm{e}-09)$ (Fig. 4A). Besides, our model had the best predictive power, compared with other clinicopathological factors such as age, gender, TNM and stage. Its area under curve (AUC) of ROC was 0.790 (Fig. 4B). The DEGGs in EGRS indicated differential expression between highand low-risk samples (Fig. 4C), and with the increase of risk score, the proportion of death cases was also increasing (Fig. 4D, E). Between different pathological groups, the expression levels of genes in EGRS also showed significant difference (Fig. 5). Contrary to CENPI, the expressions of DNAJC5B in elderly patients $(>65$ years old) were higher than those in younger patients $(<=65$ years old) (Figure 5A, B). The HMMR, RAD51AP1 were highly expressed in men than in women (Figure 5 D, E). ZNF322 was related to T stage, and its expressions in T3-4 were significantly lower than those in T1-2 (Figure 5G). The CENPI, DNAJC5B, HMMR, RAD51AP1 and ZNF322 were correlated to N stage. Except for ZNF322, other genes were highly expressed in N1-2 stage (Figure 5I-L). The expressions of ADAMTS12 in M1 stage were lower than those in M0 stage (Figure 5N). The expressions of DNAJC5B, HMMR, IGF2BP1, IGF2BP3, RAD51AP1, ZNF322 were correlated to the pathological stage. Except for ZNF322 and DNAJC5B, the expressions of other genes in stage III-IV were significantly higher than those in stage I-II (Figure 5P-U). More importantly, the risk scores in the advanced LUAD were significantly higher than those in the early stage (Figure 5H, M and V), although its distribution had no statistical difference in some pathological statuses such as age, gender and tumor metastasis (Figure 5C, F and O).

The univariate and multivariate Cox analyses suggested the risk score was an independent prognostic factor (Fig. 6A, B). The pan-cancer prognostic analysis also indicated the 11 DEGGs had prognostic value in some cancer types. Likewise, different prognostic effects based on cancer type were observed in some DEGGs (the detail results were packaged into a Supplementary file). In addition, the predictive power of the EGRS was further validated in four GEO testing datasets. Consistent with the training set, our model also stratified the samples in the testing sets into lowrisk and high-risk groups, and the low-risk patients had better outcomes (Figure $6 \mathrm{C}-\mathrm{F}$ ). The AUC results also showed that our model had stable predictive ability. The AUC in four independent GEO datasets were 0.726 (GSE30219), 0.764 (GSE50081), 0.657 (GSE37745) and 0.668 
222

223

224

225

226

227

228

229

230

231

232

233

234

235

236

237

238

239

240

241

242

243

244

245

246

247

248

249

250

251

252

253

254

255

256

257

258

259

260

(GSE72094) (Fig. 6G-J).

\section{Construction and evaluation of a nomogram}

In order to more straightforwardly predict the prognosis of LUAD patients, a nomogram was constructed (Fig. 7A). According to the expression level of 11 genes in an individual patient, a numeric value was calculated to directly predict the 1-, 3-year and 5-year prognostic survival rate. The calibration plots showed that the predictive values of OS probabilities in 1-, 3-, and 5-year fit well with the observation values, suggesting that the nomogram is suitable for predicting the prognostic survival rate of LUAD patients (Figs. 7B, 7C, 7D).

\section{Discussion}

Although the data published recently on lung cancer in the United States are encouraging, lung cancer is still a malignant tumor with high morbidity and mortality in the world(Siegel et al. 2020; Sung et al. 2021). In China, the incidence of lung cancer ranks the first and the second among men and women, respectively, and it is still the malignant disease with the highest mortality rate(Zhang et al. 2020b). The lung adenocarcinoma is the most common pathological subtype of non-small cells lung cancer (NSCLC), which accounts for about $40 \%$ of all lung cancer cases(Kleczko et al. 2019). Benefiting from carrying a higher proportion of actionable mutations, lung adenocarcinoma has more treatment options than other subtypes of lung cancer, however, its five-year overall survival rate remains unsatisfactory(Zhang et al. 2019b). Accumulating evidence indicates that patients with early stage of disease have a better prognosis, so finding more effective early diagnostic biomarkers or therapeutic targets is important for developing more effective treatment regimens, prolonging patient's survival time and improving their quality of life.

The various types of human cells are derived from a single fertilized egg cell through differentiation mechanisms that we do not fully understand(McKenna \& Gagnon 2019). In this process, specific types of genes in differentiating cells are expressed in a well-organized spatiotemporal sequence under the cues of various internal and external factors, and finally generate tissue cells with distinct morphology, function and gene expression profiles(Trott \& Martinez Arias 2013). Germline cell-specific genes are a class of genes that are expressed only in germ cells. Accumulating evidence shows that tumor cells and germ cells have similar behaviors, so it is speculated that tumor cells may adopt certain similar regulatory mechanisms in the process of tumorigenesis and development(Bruggeman et al. 2018; Simpson et al. 2005). Indeed, followup studies have detected multiple germ cell-specific genes in different tumor tissues(Erenpreisa \& Cragg 2010; McFarlane \& Wakeman 2017; Whitehurst 2014). The products of these genes are regarded as ideal immunotherapy targets due to their restricted range of expression and have attracted more and more attention.

Currently, Bruggeman et al. identified 672 true embryonic germ cell-specific genes by removing contaminated somatic genes(Bruggeman et al. 2020). However, the expression status and their prognostic value of these genes have not been fully studied in lung adenocarcinoma. To take these issues, we systematically studied the expression levels of the above-mentioned 672 
261 genes in the TCGA LUAD cohort, evaluated their correlation with the prognosis of LUAD 262 patients, and finally constructed and validated a prognostic model consisting of 11 DEGGs. Our 263 model can well stratify the patients into high- and low-risk groups and robustly predict the 264 outcomes of patients in both training and testing sets. Among the 11 DEGGs, ZNF322 (also known 265 as ZNF322A, ZNF388 or ZNF489) is a member of the zinc finger transcription factor family, and 266 may act as a positive regulator in MAPK signaling pathway(Li et al. 2004). Recent studies have 267 shown that the overexpression of ZNF322 is related to the oncogenesis of many tumors including 268 lung cancer through a variety of mechanisms, such as directly suppressing the expression of c269 Myc to promote cell stemness or up-regulating the transcription of $\alpha$-adducin (ADD1) and cyclin 270 D1 (CCND1) to promote tumor growth and metastasis(Jen et al. 2017; Jen et al. 2019; Liao et al. 2017; Williams et al. 2014). DNA binding protein RAD51AP1 is a component of DNA damage repair system, which plays an important role in RAD51-mediated homologous recombination(Pires et al. 2017). Further studies indicates that overexpression of RAD51AP1 promotes the proliferation of lung cancer cells and correlates to poor prognosis(Chudasama et al. 2018; Li et al. 2018b; Wu et al. 2019). HMMR coding a 724 amino acids protein, which may associate with the motility of cancer cells(Sankaran et al. 2012). Quite a few of studies had shown that the highly expression of HMMR is closely related to the prognosis of LUAD patients, and it may promote the progression of lung adenocarcinoma by regulating the metabolic state of tumor cells(He \& Zuo 2019; Jiao et al. 2020; Li et al. 2020a; Li et al. 2020c; Liu et al. 2019; Man et al. 2014; Shen et al. 2019; Stevens et al. 2017; Zhang et al. 2019a; Zhou et al. 2015). The ADAMTS12 is a novel anti-tumor metalloprotease, and the expression is epigenetically silenced in tumor cells(Li et al. 2018a; Moncada-Pazos et al. 2009). A bulk of studies suggest the overexpression of IGF2BP1 and IGF2BP3 facilitate the progress of lung cancer, and relate to the prognosis of LUAD patients(Beljan Perak et al. 2012; Gong et al. 2016; Guo et al. 2021; Huang et al. 2019; Kato et al. 2007; Li \& Zhan 2020; Li et al. 2020b; Mizutani et al. 2016; Müller et al. 2019; Ohdaira et al. 2012; Wang et al. 2020; Xueqing et al. 2020; Zhang et al. 2020a). E2F7 plays an important physiological role in embryonic development and cell cycle regulation (Park et al. 2015). Recent researches show the various non-coding RNA can influence the progress of lung cancer by regulating the expression of E2F7(Liang et al. 2018; Liu et al. 2020; Wang et al. 2021; Yuan et al. 2021). NANOS1 was up-regulated in lung cancer, and promoted the progress of tumor progress(Bonnomet et al. 2008; De Keuckelaere et al. 2018). RAD54L is also a potential prognostic biomarker of NSCLC(Tu et al. 2021; Zheng et al. 2021). Literature retrieval showed that the relation of LUAD and DEGGs DNAJC5B, CENPI has not been reported so far. In addition, pan-cancer expression and prognosis analyses also indicated that 11 DEGGs showed differential expression and prognostic value in a variety of tumors, suggesting that these DEGGs may also play a role in the development of tumors other than lung adenocarcinoma, which needed to be validated by further functional experiments.

Although we constructed and validated an 11-gene prognostic model, the present study still has several limitations: First, the number of samples in training set is not large enough; secondly, due to tumor heterogeneity and individual differences among LUAD patients, the generalizing abilities of the model need further improvement. Therefore, further model optimization and 
302 validation using LUAD cohort with larger samples are needed. In addition, the results of 303 bioinformatics analysis did not carry out related experimental verifications. Therefore, whether the 304 genes in the model play a functional role in the progress of LUAD remains to be resolved. These 305 issues mentioned above will be the main content of our follow-up studies.

\section{Conclusion}

In summary, we used LUAD transcriptome data to identify DEGGs. From the DEGGs, an 11gene signature and a prognostic nomogram were constructed and validated for predicting the outcomes of LUAD patients. Further studies on these genes will provide a new insight into the potential relationship between tumor microenvironment and LUAD prognosis.

\section{Acknowledgments}

Not applicable

\section{Reference}

Almeida LG, Sakabe NJ, deOliveira AR, Silva MC, Mundstein AS, Cohen T, Chen YT, Chua R, Gurung S, Gnjatic S, Jungbluth AA, Caballero OL, Bairoch A, Kiesler E, White SL, Simpson AJ, Old LJ, Camargo AA, and Vasconcelos AT. 2009. CTdatabase: a knowledge-base of high-throughput and curated data on cancer-testis antigens. Nucleic Acids Res 37:D816-819. 10.1093/nar/gkn673

Beljan Perak R, Durdov MG, Capkun V, Ivcevic V, Pavlovic A, Soljic V, and Peric M. 2012. IMP3 can predict aggressive behaviour of lung adenocarcinoma. Diagn Pathol 7:165. 10.1186/1746-1596-7-165

Benjamini Y, and Hochberg Y. 1995. Controlling the false discovery rate: a practical and powerful approach to multiple testing. Journal of the Royal statistical society: series B 57:289-300.

Boël P, Wildmann C, Sensi ML, Brasseur R, Renauld JC, Coulie P, Boon T, and van der Bruggen P. 1995. BAGE: a new gene encoding an antigen recognized on human melanomas by cytolytic $\mathrm{T}$ lymphocytes. Immunity 2:167-175. 10.1016/s1074-7613(95)80053-0

Bonnomet A, Polette M, Strumane K, Gilles C, Dalstein V, Kileztky C, Berx G, van Roy F, Birembaut P, and Nawrocki-Raby B. 2008. The E-cadherin-repressed hNanos1 gene induces tumor cell invasion by upregulating MT1-MMP expression. Oncogene 27:3692-3699. 10.1038/sj.onc.1211035

Bruggeman JW, Irie N, Lodder P, van Pelt AMM, Koster J, and Hamer G. 2020. Tumors Widely Express Hundreds of Embryonic Germline Genes. Cancers (Basel) 12. 10.3390/cancers 12123812

Bruggeman JW, Koster J, Lodder P, Repping S, and Hamer G. 2018. Massive expression of germ cell-specific genes is a hallmark of cancer and a potential target for novel treatment development. Oncogene 37:5694-5700. 10.1038/s41388-018-0357-2

Chen YT, Scanlan MJ, Sahin U, Türeci O, Gure AO, Tsang S, Williamson B, Stockert E, Pfreundschuh M, and Old LJ. 1997. A testicular antigen aberrantly expressed in human cancers detected by autologous antibody screening. Proc Natl Acad Sci U S A 94:1914-1918. 10.1073/pnas.94.5.1914

Chen Z, Fillmore CM, Hammerman PS, Kim CF, and Wong KK. 2014. Non-small-cell lung cancers: a heterogeneous set of diseases. Nat Rev Cancer 14:535-546. 10.1038/nrc3775 
Chudasama D, Bo V, Hall M, Anikin V, Jeyaneethi J, Gregory J, Pados G, Tucker A, Harvey A, Pink R, and Karteris E. 2018. Identification of cancer biomarkers of prognostic value using specific gene regulatory networks (GRN): a novel role of RAD51AP1 for ovarian and lung cancers. Carcinogenesis 39:407-417. $10.1093 /$ carcin/bgx 122

Davis S, and Meltzer PS. 2007. GEOquery: a bridge between the Gene Expression Omnibus (GEO) and BioConductor. Bioinformatics 23:1846-1847. 10.1093/bioinformatics/btm254

De Backer O, Arden KC, Boretti M, Vantomme V, De Smet C, Czekay S, Viars CS, De Plaen E, Brasseur F, Chomez P, Van den Eynde B, Boon T, and van der Bruggen P. 1999. Characterization of the GAGE genes that are expressed in various human cancers and in normal testis. Cancer Res 59:3157-3165.

De Keuckelaere E, Hulpiau P, Saeys Y, Berx G, and van Roy F. 2018. Nanos genes and their role in development and beyond. Cell Mol Life Sci 75:1929-1946. 10.1007/s00018-018-2766-3

Erenpreisa J, and Cragg MS. 2010. MOS, aneuploidy and the ploidy cycle of cancer cells. Oncogene 29:5447-5451. 10.1038/onc. 2010.310

Friedman J, Hastie T, and Tibshirani R. 2010. Regularization Paths for Generalized Linear Models via Coordinate Descent. J Stat Softw 33:1-22.

Gong F, Ren P, Zhang Y, Jiang J, and Zhang H. 2016. MicroRNAs-491-5p suppresses cell proliferation and invasion by inhibiting IGF2BP1 in non-small cell lung cancer. Am J Transl Res 8:485-495.

Guo W, Huai Q, Wan H, Guo L, Song P, Gao S, and He J. 2021. Prognostic Impact of IGF2BP3 Expression in Patients with Surgically Resected Lung Adenocarcinoma. DNA Cell Biol 40:316-331. 10.1089/dna.2020.6136

He R, and Zuo S. 2019. A Robust 8-Gene Prognostic Signature for Early-Stage Non-small Cell Lung Cancer. Front Oncol 9:693. 10.3389/fonc.2019.00693

Herbst RS, Morgensztern D, and Boshoff C. 2018. The biology and management of non-small cell lung cancer. Nature 553:446-454. 10.1038/nature25183

Huang H, Wang D, Guo W, Zhuang X, and He Y. 2019. Correlated low IGF2BP1 and FOXM1 expression predicts a good prognosis in lung adenocarcinoma. Pathol Res Pract 215:152433. 10.1016/j.prp.2019.152433

Jen J, Lin LL, Lo FY, Chen HT, Liao SY, Tang YA, Su WC, Salgia R, Hsu CL, Huang HC, Juan HF, and Wang YC. 2017. Oncoprotein ZNF322A transcriptionally deregulates alpha-adducin, cyclin D1 and p53 to promote tumor growth and metastasis in lung cancer. Oncogene 36:5219. 10.1038/onc.2017.203

Jen J, Liu CY, Chen YT, Wu LT, Shieh YC, Lai WW, and Wang YC. 2019. Oncogenic zinc finger protein ZNF322A promotes stem cell-like properties in lung cancer through transcriptional suppression of c-Myc expression. Cell Death Differ 26:1283-1298. 10.1038/s41418-018-0204-6

Jiao Z, Yu A, He X, Xuan Y, Zhang H, Wang G, Shi M, and Wang T. 2020. Bioinformatics analysis to determine the prognostic value and prospective pathway signaling of miR-126 in non-small cell lung cancer. Ann Transl Med 8:1639. 10.21037/atm-20-7520

Kato T, Hayama S, Yamabuki T, Ishikawa N, Miyamoto M, Ito T, Tsuchiya E, Kondo S, Nakamura Y, and Daigo Y. 2007. Increased expression of insulin-like growth factor-II messenger RNA-binding protein 1 is associated with tumor progression in patients with lung cancer. Clin Cancer Res 13:434-442. 10.1158/1078-0432.Ccr06-1297

Kho AT, Zhao Q, Cai Z, Butte AJ, Kim JY, Pomeroy SL, Rowitch DH, and Kohane IS. 2004. Conserved mechanisms across development and tumorigenesis revealed by a mouse development perspective of human cancers. Genes Dev 18:629-640. 10.1101/gad.1182504 
382

383

384

385

386

387

388

389

390

391

392

393

394

395

396

397

398

399

400

401

402

403

404

405

406

407

408

409

410

411

412

413

414

415

416

417

418

419

420

421

422

Kleczko EK, Kwak JW, Schenk EL, and Nemenoff RA. 2019. Targeting the Complement Pathway as a Therapeutic Strategy in Lung Cancer. Front Immunol 10:954. 10.3389/fimmu.2019.00954

Lepourcelet M, Tou L, Cai L, Sawada J, Lazar AJ, Glickman JN, Williamson JA, Everett AD, Redston M, Fox EA, Nakatani Y, and Shivdasani RA. 2005. Insights into developmental mechanisms and cancers in the mammalian intestine derived from serial analysis of gene expression and study of the hepatoma-derived growth factor (HDGF). Development 132:415-427. 10.1242/dev.01579

Li J, Liao Y, Huang J, Sun Y, Chen H, Chen C, Li S, and Yang Z. 2018a. Epigenetic silencing of ADAMTS5 is associated with increased invasiveness and poor survival in patients with colorectal cancer. J Cancer Res Clin Oncol 144:215-227. 10.1007/s00432-017-2545-9

Li N, and Zhan X. 2020. Identification of pathology-specific regulators of m(6)A RNA modification to optimize lung cancer management in the context of predictive, preventive, and personalized medicine. Epma j 11:485-504. 10.1007/s13167-020-00220-3

Li S, Xuan Y, Gao B, Sun X, Miao S, Lu T, Wang Y, and Jiao W. 2018b. Identification of an eight-gene prognostic signature for lung adenocarcinoma. Cancer Manag Res 10:3383-3392. 10.2147/cmar.S173941

Li W, Pan T, Jiang W, and Zhao H. 2020a. HCG18/miR-34a-5p/HMMR axis accelerates the progression of lung adenocarcinoma. Biomed Pharmacother 129:110217. 10.1016/j.biopha.2020.110217

Li Y, Gu J, Xu F, Zhu Q, Chen Y, Ge D, and Lu C. 2020b. Molecular characterization, biological function, tumor microenvironment association and clinical significance of m6A regulators in lung adenocarcinoma. Brief Bioinform. 10.1093/bib/bbaa225

Li Y, Wang Y, Zhang C, Yuan W, Wang J, Zhu C, Chen L, Huang W, Zeng W, Wu X, and Liu M. 2004. ZNF322, a novel human $\mathrm{C} 2 \mathrm{H} 2$ Kruppel-like zinc-finger protein, regulates transcriptional activation in MAPK signaling pathways. Biochem Biophys Res Commun 325:1383-1392. 10.1016/j.bbrc.2004.10.183

Li Z, Qi F, and Li F. 2020c. Establishment of a Gene Signature to Predict Prognosis for Patients with Lung Adenocarcinoma. Int J Mol Sci 21. 10.3390/ijms21228479

Liang R, Xiao G, Wang M, Li X, Li Y, Hui Z, Sun X, Qin S, Zhang B, Du N, Liu D, and Ren H. 2018. SNHG6 functions as a competing endogenous RNA to regulate E2F7 expression by sponging miR-26a-5p in lung adenocarcinoma. Biomed Pharmacother 107:1434-1446. 10.1016/j.biopha.2018.08.099

Liao SY, Chiang CW, Hsu CH, Chen YT, Jen J, Juan HF, Lai WW, and Wang YC. 2017. CK1 $\delta / G S K 3 \beta / F B X W 7 \alpha$ axis promotes degradation of the ZNF322A oncoprotein to suppress lung cancer progression. Oncogene 36:5722-5733. 10.1038/onc.2017.168

Liu C, Li Y, Wei M, Zhao L, Yu Y, and Li G. 2019. Identification of a novel glycolysis-related gene signature that can predict the survival of patients with lung adenocarcinoma. Cell Cycle 18:568-579. $10.1080 / 15384101.2019 .1578146$

Liu P, Wang M, Tang W, Li G, and Gong N. 2020. Circ_SATB2 Attenuates the Anti-Tumor Role of Celastrol in NonSmall-Cell Lung Carcinoma Through Targeting miR-33a-5p/E2F7 Axis. Onco Targets Ther 13:1189911912. 10.2147/ott.S279434

Man Y, Cao J, Jin S, Xu G, Pan B, Shang L, Che D, Yu Q, and Yu Y. 2014. Newly identified biomarkers for detecting circulating tumor cells in lung adenocarcinoma. Tohoku J Exp Med 234:29-40. 10.1620/tjem.234.29

McFarlane RJ, and Wakeman JA. 2017. Meiosis-like Functions in Oncogenesis: A New View of Cancer. Cancer Res 77:5712-5716. 10.1158/0008-5472.CAN-17-1535

McKenna A, and Gagnon JA. 2019. Recording development with single cell dynamic lineage tracing. Development

Peer) reviewing PDF | (2021:06:61915:1:1:NEW 13 Aug 2021) 
146. $10.1242 /$ dev. 169730

Mizutani R, Imamachi N, Suzuki Y, Yoshida H, Tochigi N, Oonishi T, Suzuki Y, and Akimitsu N. 2016. Oncofetal protein IGF2BP3 facilitates the activity of proto-oncogene protein eIF4E through the destabilization of EIF4E-BP2 mRNA. Oncogene 35:3495-3502. 10.1038/onc.2015.410

Moncada-Pazos A, Obaya AJ, Fraga MF, Viloria CG, Capellá G, Gausachs M, Esteller M, López-Otín C, and Cal S. 2009. The ADAMTS12 metalloprotease gene is epigenetically silenced in tumor cells and transcriptionally activated in the stroma during progression of colon cancer. J Cell Sci 122:2906-2913. 10.1242/jcs.050468

Müller S, Glaß M, Singh AK, Haase J, Bley N, Fuchs T, Lederer M, Dahl A, Huang H, Chen J, Posern G, and Hüttelmaier S. 2019. IGF2BP1 promotes SRF-dependent transcription in cancer in a m6A- and miRNAdependent manner. Nucleic Acids Res 47:375-390. 10.1093/nar/gky1012

Ohdaira H, Sekiguchi M, Miyata K, and Yoshida K. 2012. MicroRNA-494 suppresses cell proliferation and induces senescence in A549 lung cancer cells. Cell Prolif 45:32-38. 10.1111/j.1365-2184.2011.00798.x

Park SA, Platt J, Lee JW, López-Giráldez F, Herbst RS, and Koo JS. 2015. E2F8 as a Novel Therapeutic Target for Lung Cancer. J Natl Cancer Inst 107. 10.1093/jnci/djv151

Pires E, Sung P, and Wiese C. 2017. Role of RAD51AP1 in homologous recombination DNA repair and carcinogenesis. DNA Repair (Amst) 59:76-81. 10.1016/j.dnarep.2017.09.008

Ritchie ME, Phipson B, Wu D, Hu Y, Law CW, Shi W, and Smyth GK. 2015. limma powers differential expression analyses for RNA-sequencing and microarray studies. Nucleic Acids Res 43:e47. 10.1093/nar/gkv007

Sankaran D, Pakala SB, Nair VS, Sirigiri DN, Cyanam D, Ha NH, Li DQ, Santhoshkumar TR, Pillai MR, and Kumar R. 2012. Mechanism of MTA1 protein overexpression-linked invasion: MTA1 regulation of hyaluronanmediated motility receptor (HMMR) expression and function. J Biol Chem 287:5483-5491. 10.1074/jbc.M111.324632

Shen S, Kong J, Qiu Y, Yang X, Wang W, and Yan L. 2019. Identification of core genes and outcomes in hepatocellular carcinoma by bioinformatics analysis. J Cell Biochem 120:10069-10081. 10.1002/jcb.28290

Siegel RL, Miller KD, and Jemal A. 2020. Cancer statistics, 2020. CA Cancer J Clin 70:7-30. 10.3322/caac.21590

Simpson AJ, Caballero OL, Jungbluth A, Chen YT, and Old LJ. 2005. Cancer/testis antigens, gametogenesis and cancer. Nat Rev Cancer 5:615-625. 10.1038/nrc1669

Stevens LE, Cheung WKC, Adua SJ, Arnal-Estapé A, Zhao M, Liu Z, Brewer K, Herbst RS, and Nguyen DX. 2017. Extracellular Matrix Receptor Expression in Subtypes of Lung Adenocarcinoma Potentiates Outgrowth of Micrometastases. Cancer Res 77:1905-1917. 10.1158/0008-5472.Can-16-1978

Sung H, Ferlay J, Siegel RL, Laversanne M, Soerjomataram I, Jemal A, and Bray F. 2021. Global cancer statistics 2020: GLOBOCAN estimates of incidence and mortality worldwide for 36 cancers in 185 countries. CA Cancer J Clin. 10.3322/caac.21660

Trott J, and Martinez Arias A. 2013. Single cell lineage analysis of mouse embryonic stem cells at the exit from pluripotency. Biol Open 2:1049-1056. 10.1242/bio.20135934

Tu Z, Chen X, Tian T, Chen G, and Huang M. 2021. Prognostic significance of epigenetic regulatory gene expression in patients with non-small-cell lung cancer. Aging (Albany NY) 13:7397-7415. 10.18632/aging.202600

Türeci O, Sahin U, Schobert I, Koslowski M, Scmitt H, Schild HJ, Stenner F, Seitz G, Rammensee HG, and Pfreundschuh M. 1996. The SSX-2 gene, which is involved in the t(X;18) translocation of synovial sarcomas, codes for the human tumor antigen HOM-MEL-40. Cancer Res 56:4766-4772.

Türeci O, Sahin U, Zwick C, Koslowski M, Seitz G, and Pfreundschuh M. 1998. Identification of a meiosis-specific 
464

465

466

467

468

469

470

471

472

473

474

475

476

477

478

479

480

481

482

483

484

485

486

487

488

489

490

491

492

493

494

495

496

497

498

499

500

501

502

503

504

protein as a member of the class of cancer/testis antigens. Proc Natl Acad Sci U S A 95:5211-5216. 10.1073/pnas.95.9.5211

van der Bruggen P, Traversari C, Chomez P, Lurquin C, De Plaen E, Van den Eynde B, Knuth A, and Boon T. 1991. A gene encoding an antigen recognized by cytolytic $\mathrm{T}$ lymphocytes on a human melanoma. Science 254:1643-1647. 10.1126/science.1840703

Wang Y, He R, and Ma L. 2020. Characterization of IncRNA-Associated ceRNA Network to Reveal Potential Prognostic Biomarkers in Lung Adenocarcinoma. Front Bioeng Biotechnol 8:266. 10.3389/fbioe.2020.00266

Wang Y, Wo Y, Lu T, Sun X, Liu A, Dong Y, Du W, Su W, Huang Z, and Jiao W. 2021. Circ-AASDH functions as the progression of early stage lung adenocarcinoma by targeting miR-140-3p to activate E2F7 expression. Transl Lung Cancer Res 10:57-70. 10.21037/tlcr-20-1062

Whitehurst AW. 2014. Cause and consequence of cancer/testis antigen activation in cancer. Annu Rev Pharmacol Toxicol 54:251-272. 10.1146/annurev-pharmtox-011112-140326

Williams KA, Lee M, Hu Y, Andreas J, Patel SJ, Zhang S, Chines P, Elkahloun A, Chandrasekharappa S, Gutkind JS, Molinolo AA, and Crawford NP. 2014. A systems genetics approach identifies CXCL14, ITGAX, and LPCAT2 as novel aggressive prostate cancer susceptibility genes. PLoS Genet 10:e1004809. 10.1371/journal.pgen.1004809

Wu Y, Wang H, Qiao L, Jin X, Dong H, and Wang Y. 2019. Silencing of RAD51AP1 suppresses epithelialmesenchymal transition and metastasis in non-small cell lung cancer. Thorac Cancer 10:1748-1763. 10.1111/1759-7714.13124

Xueqing H, Jun Z, Yueqiang J, Xin L, Liya H, Yuanyuan F, Yuting Z, Hao Z, Hua W, Jian L, and Tiejun Y. 2020. IGF2BP3 May Contributes to Lung Tumorigenesis by Regulating the Alternative Splicing of PKM. Front Bioeng Biotechnol 8:679. 10.3389/fbioe.2020.00679

Yang CY, Yang JC, and Yang PC. 2020. Precision Management of Advanced Non-Small Cell Lung Cancer. Annu Rev Med 71:117-136. 10.1146/annurev-med-051718-013524

Yu G, Wang LG, Han Y, and He QY. 2012. clusterProfiler: an R package for comparing biological themes among gene clusters. Omics 16:284-287. 10.1089/omi.2011.0118

Yuan Y, Zhou X, Kang Y, Kuang H, Peng Q, Zhang B, Liu X, and Zhang M. 2021. Circ-CCS is identified as a cancerpromoting circRNA in lung cancer partly by regulating the miR-383/E2F7 axis. Life Sci 267:118955. $10.1016 / j .1 f s .2020 .118955$

Yue C, Ma H, and Zhou Y. 2019. Identification of prognostic gene signature associated with microenvironment of lung adenocarcinoma. PeerJ 7:e8128. 10.7717/peerj.8128

Zhang J, Luo W, Chi X, Zhang L, Ren Q, Wang H, and Zhang W. 2020a. IGF2BP1 silencing inhibits proliferation and induces apoptosis of high glucose-induced non-small cell lung cancer cells by regulating Netrin-1. Arch Biochem Biophys 693:108581. 10.1016/j.abb.2020.108581

Zhang L, Zhang Z, and Yu Z. 2019a. Identification of a novel glycolysis-related gene signature for predicting metastasis and survival in patients with lung adenocarcinoma. J Transl Med 17:423. 10.1186/s12967-01902173-2

Zhang S, Sun K, Zheng R, Zeng H, Wang S, Chen R, Wei W, and He J. 2020b. Cancer incidence and mortality in China, 2015. Journal of the National Cancer Center. 10.1016/j.jncc.2020.12.001

Zhang Y, Wang LF, Gao JH, Li L, Jiang P, Lv X, Yu LX, Yang J, Li RT, and Liu BR. 2019b. Clinical significance of epithelial-mesenchymal transition-related molecules in lung adenocarcinoma. Curr Oncol 26:e121-e127.

Peer) reviewing PDF | (2021:06:61915:1:1:NEW 13 Aug 2021) 
$10.3747 /$ co.26.4471

506

Zheng S, Yao L, Li F, Huang L, Yu Y, Lin Z, Li H, Xia J, Lanuti M, and Zhou H. 2021. Homologous recombination repair rathway and RAD54L in early-stage lung adenocarcinoma. PeerJ 9:e10680. 10.7717/peerj.10680

Zhou H, Wang L, Huang J, Jiang M, Zhang X, Zhang L, Wang Y, Jiang Z, and Zhang Z. 2015. High EGFR_1 Inside509 Out Activated Inflammation-Induced Motility through SLC2A1-CCNB2-HMMR-KIF11-NUSAP1-PRC1UBE2C. J Cancer 6:519-524. 10.7150/jca.11404 


\section{Figure 1}

The differentially expressed embryonic germline genes ( DEGGs ) between LUAD and normal tissues.

(A) The volcano plot of DEGGs. The red and green dots represent the up-regulated and downregulated DEGGs, and the black dots represent the embryonic germline genes that do not meet the selection criteria. (B) The heatmap of the representative DEGGs. $\mathrm{N}$ and T represent the normal and LUAD samples, respectively. The color of scale bar (from green to red) indicates the expression status of DEGGs (from lowly expression to highly expression). (C) The boxplot of the representative DEGGs. $\mathrm{P}<0.05$ was considered as statistical significance. LUAD: Lung adenocarcinoma; FDR: False Discovery Rate.
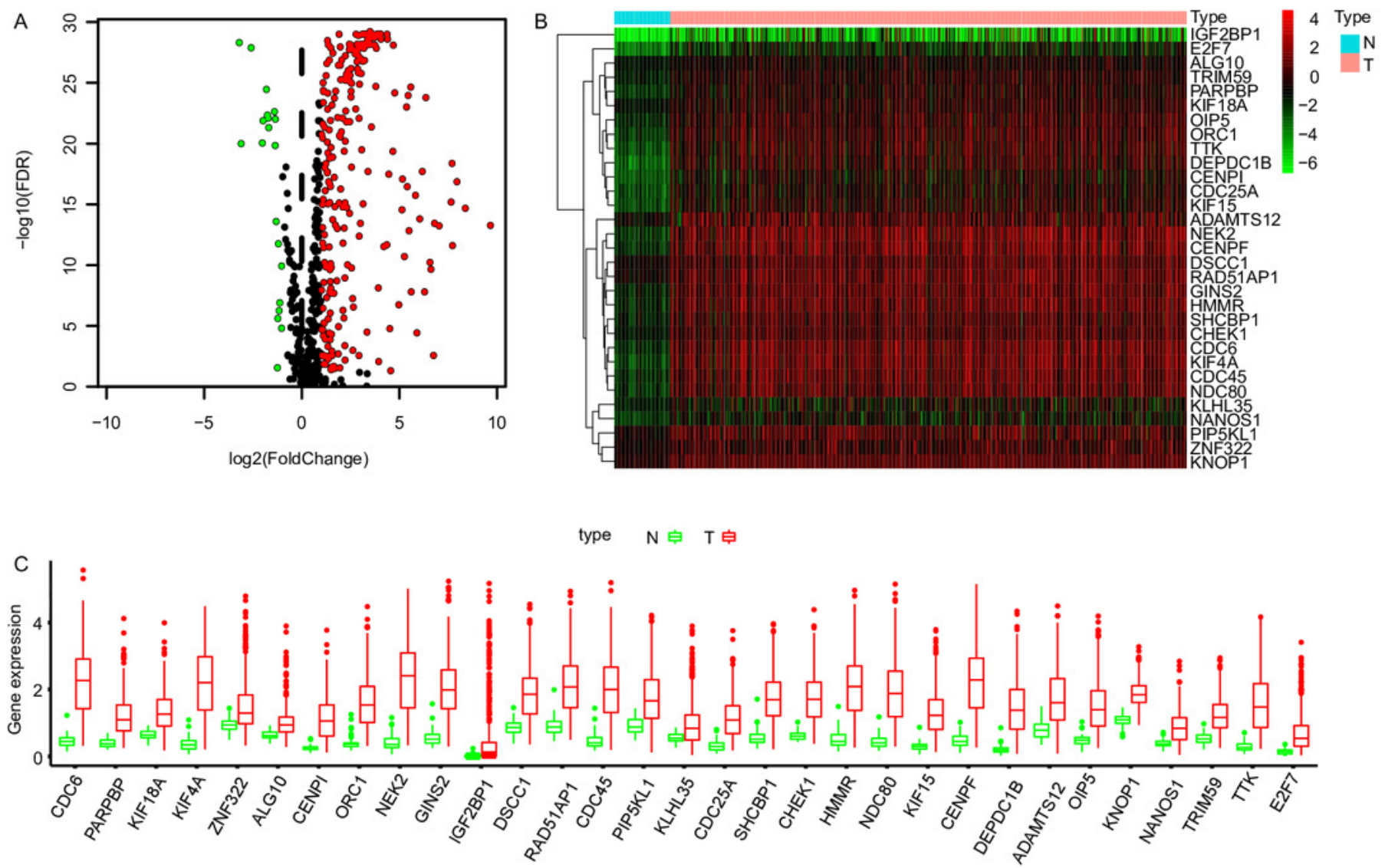


\section{Figure 2}

The GO and KEGG enrichment analysis of DEGGs.

(A, C): The bubble plot of GO and KEGG pathway analysis of DEGGs, respectively. The color scale represents large (blue) to small (red) p.adjust values. The size of the black dots represents the number of DEGGs enriched in the corresponding terms or pathways. $(B, D)$ : The heat plot of GO and KEGG pathway analysis of DEGGs, respectively. The color depth represents the expression status of DEGGs, and the length of the bar represents the number of DEGGs enriched in the corresponding terms or pathways. BP: Biological Process; CC: Cellular Component and MF: Molecular Function; GO: Gene Ontology; KEGG: Kyoto Encyclopedia of Genes and Genomes; logFC: log2FoldChange; DEGGs: differentially expressed embryonic germline genes.
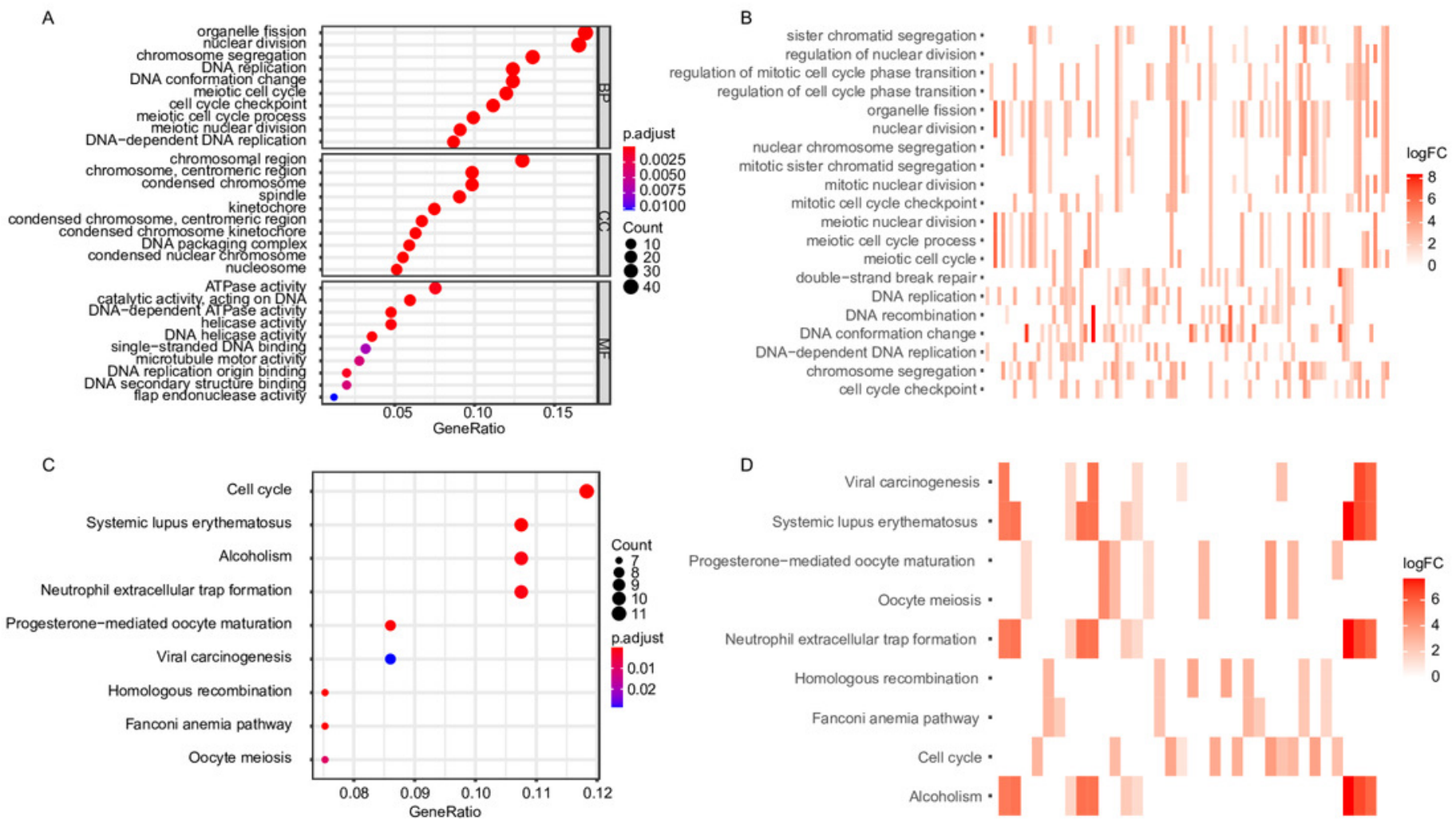
Figure 3

The identification of the DEGGs with prognostic value.

(A): The forest plot of the representative prognostic DEGGs screened out by univariate Cox proportional hazards regression. The red rectangles represent hazard factors, and green rectangles represent protective factors. (B): Lasso co-efficient profiles of prognostic DEGGs by optimal lambda. (C): The partial likelihood deviance plot presented the minimum number corresponds to the covariates used for multivariate Cox analysis. DEGGs: differentially expressed embryonic germline genes; Lasso: least absolute shrinkage and selection operator.

A

\begin{tabular}{lrc} 
& & \\
CDC6 & pvalue & Hazard ratio \\
PARPBP & 0.010 & $1.225(1.050-1.429)$ \\
KIF18A & 0.027 & $1.302(1.031-1.644)$ \\
KIF4A & $<0.001$ & $1.694(1.317-2.178)$ \\
ZNF322 & $<0.001$ & $1.332(1.141-1.555)$ \\
ALG10 & 0.037 & $0.768(0.599-0.984)$ \\
CENPI & 0.005 & $1.742(1.185-2.562)$ \\
ORC1 & 0.020 & $1.340(1.047-1.713)$ \\
NEK2 & 0.012 & $1.280(1.056-1.552)$ \\
GINS2 & $<0.001$ & $1.332(1.145-1.549)$ \\
IGF2BP1 & 0.007 & $1.269(1.066-1.511)$ \\
DSCC1 & $<0.001$ & $1.359(1.201-1.539)$ \\
RAD51AP1 & 0.007 & $1.290(1.072-1.553)$ \\
CDC45 & 0.019 & $1.220(1.033-1.440)$ \\
PIP5KL1 & 0.021 & $1.192(1.026-1.384)$ \\
KLHL35 & 0.006 & $0.762(0.627-0.925)$ \\
CDC25A & 0.026 & $0.758(0.594-0.967)$ \\
SHCBP1 & 0.003 & $1.415(1.122-1.784)$ \\
CHEK1 & $<0.001$ & $1.424(1.167-1.739)$ \\
HMMR & $<0.001$ & $1.451(1.191-1.767)$ \\
NDC80 & $<0.001$ & $1.474(1.242-1.749)$ \\
KIF15 & $<0.001$ & $1.320(1.129-1.542)$ \\
CENPF & 0.032 & $1.265(1.021-1.569)$ \\
DEPDC1B & $<0.001$ & $1.309(1.124-1.523)$ \\
ADAMTS12 & $<0.001$ & $1.407(1.175-1.684)$ \\
OIP5 & 0.035 & $1.202(1.013-1.428)$ \\
KNOP1 & $<0.001$ & $1.411(1.180-1.686)$ \\
NANOS1 & 0.032 & $1.520(1.036-2.232)$ \\
TRIM59 & 0.013 & $0.630(0.438-0.907)$ \\
TTK & 0.004 & $1.585(1.159-2.166)$ \\
E2F7 & 0.009 & $1.255(1.059-1.489)$ \\
& $<0.001$ & $1.878(1.485-2.376)$ \\
\hline
\end{tabular}

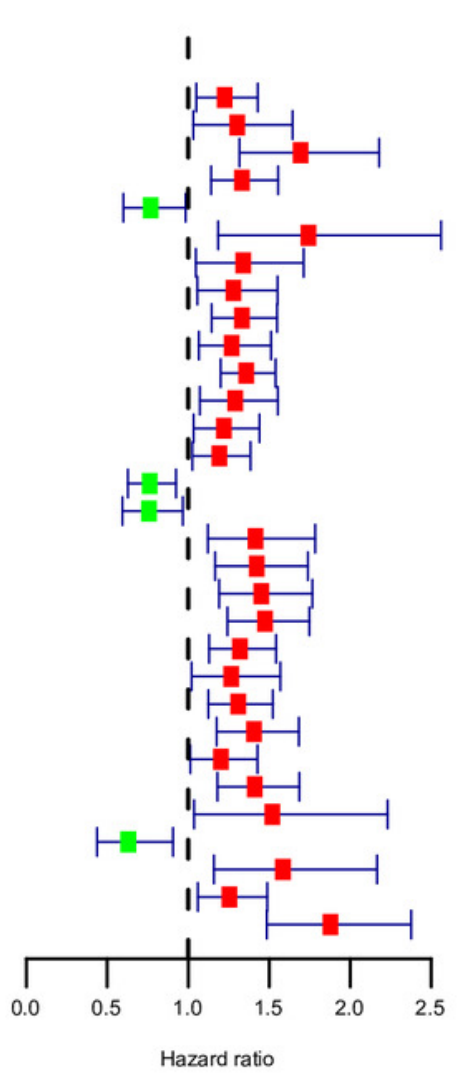

B

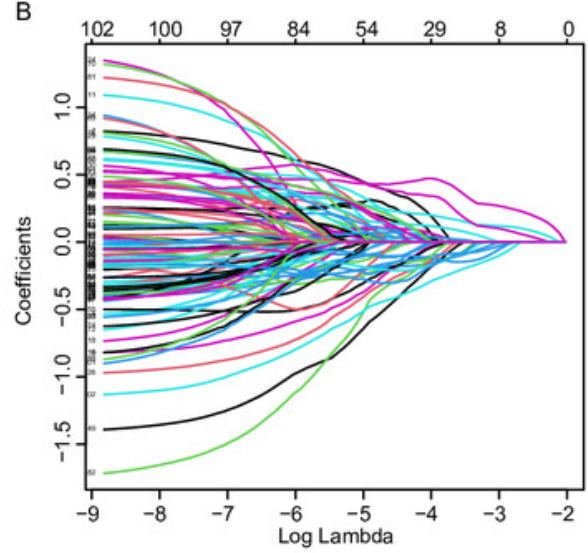

C $\quad 1021011019794897559463621128333$

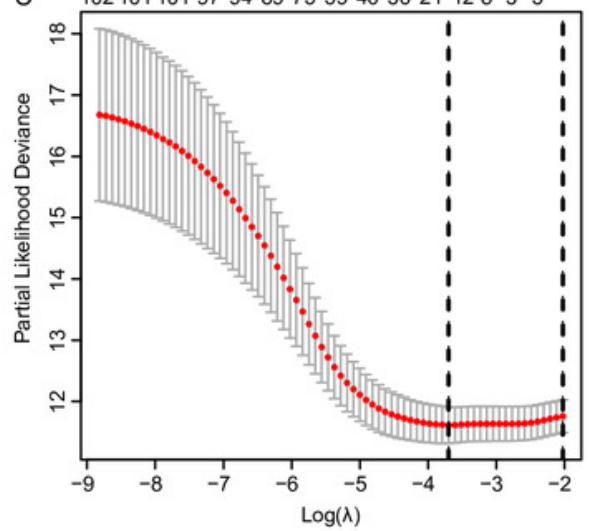




\section{Figure 4}

The construction of embryonic germline gene related prognostic model.

(A): Survival curve of low- and high-risk groups of training set stratified by EGRS. (B): The multi-ROC curve of EGRS and other clinicopathological factors for survival prediction in training set. $\mathrm{P}<0.05$ was considered as statistical significance. (C): The expression status of 11 risk DEGGs in the high-risk (cyan) and low-risk (light red) groups of LUAD patients in training set. (D): The risk score distribution of low-risk (green) and high-risk (red) groups of LUAD patients in training set. (E): The scatter plot of survival status of LUAD patients in training set. The red dots represent the dead patients, and the green dots represent the alive patients. LUAD: Lung adenocarcinoma; DEGGs: differentially expressed embryonic germline genes; EGRS: embryonic germline gene related signature ; ROC: Receiver Operating Characteristic; AUC: Area Under Curve. 


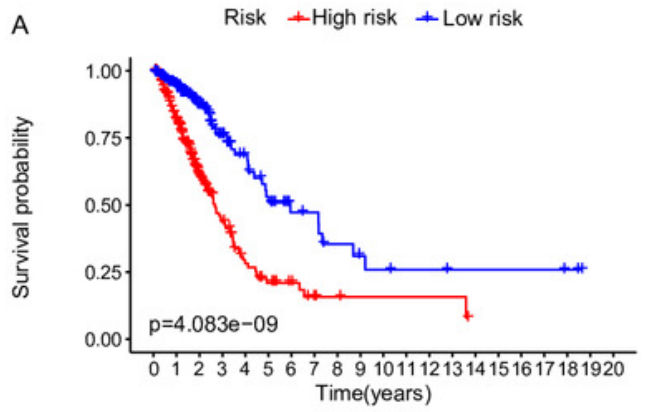

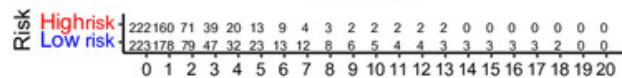
Time(years)

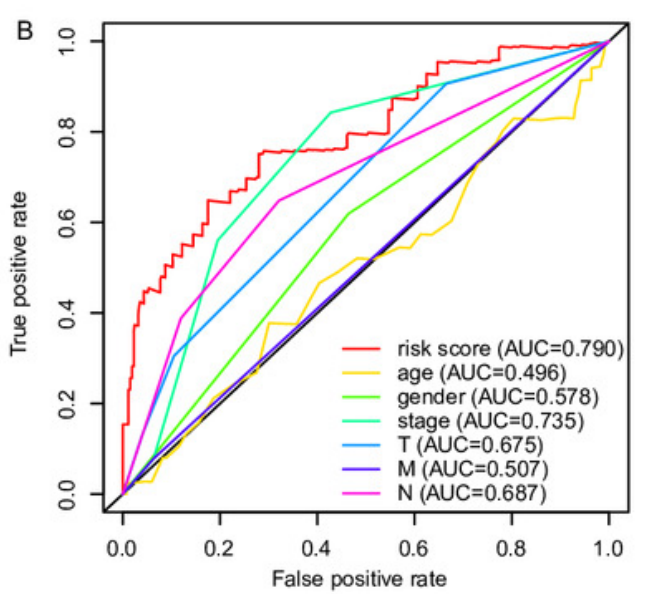

C

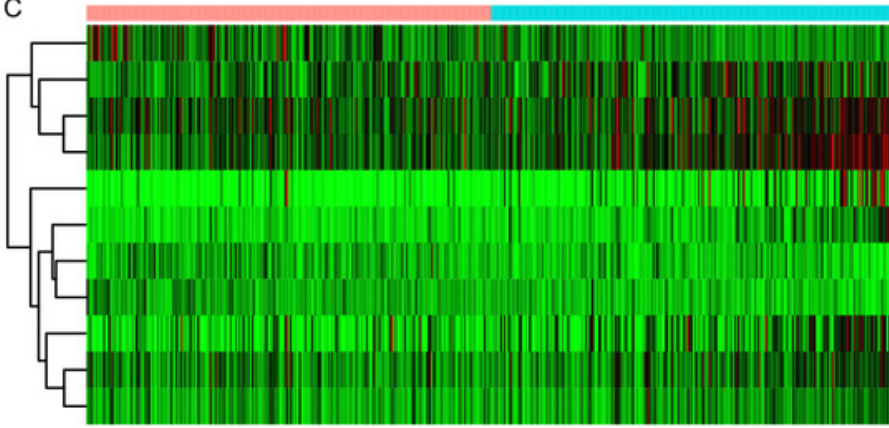

type type

ZNF322 high ADAMTS12 low RAD51AP1 HMMR IGF2BP1

E2F7

DNAJC5B

NANOS1

IGF2BP3

RAD54L

CENPI
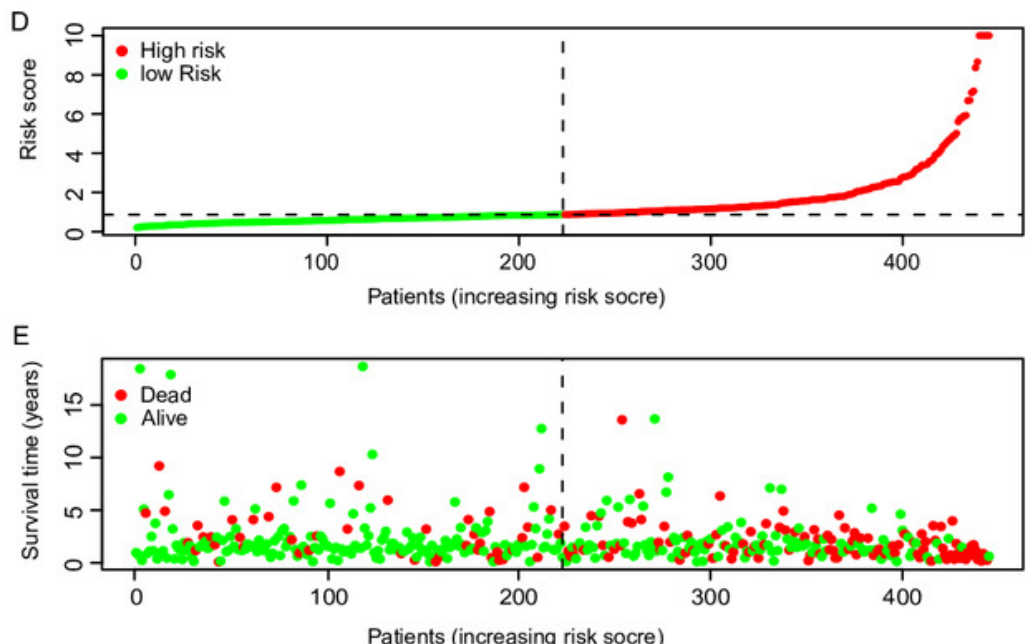
Figure 5

The distributions of ERGS genes' expressions or risk score to pathological status in training set.

(A-C): age, (D-F): gender, (G, H): T, (I-M): N, (N, O): M and (P-V): stage. EGRS: embryonic germline gene related signature.
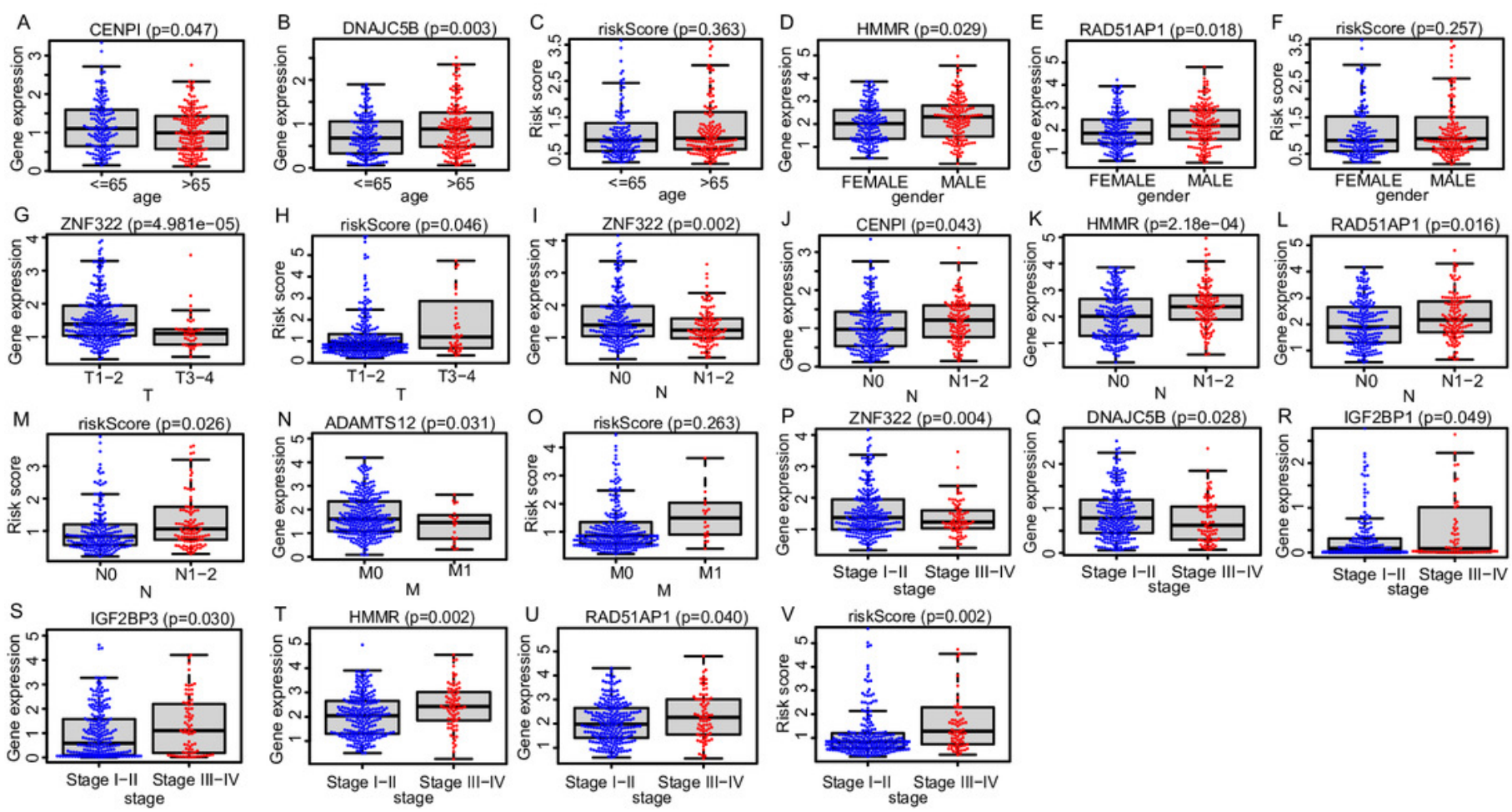


\section{Figure 6}

The validation of embryonic germline gene related prognostic model.

(A): The prognostic effect analyses of EGRS and commonly used prognostic factors using univariate Cox regression model. (B): The independent prognostic effect analyses of EGRS and commonly used prognostic factors using multivariate Cox regression model. KaplanMeier survival curves showing overall survival outcomes of GSE30219 (C), GSE50081 (D), GSE37745 (E) and GSE72094 (F) according to high-risk and low-risk patients stratified by EGRS. The ROC analysis of GSE30219 (G), GSE50081 (H), GSE37745 (I) and GSE72094 (J) for survival prediction by the EGRS. ROC: Receiver Operating Characteristic; AUC: Area Under Curve.
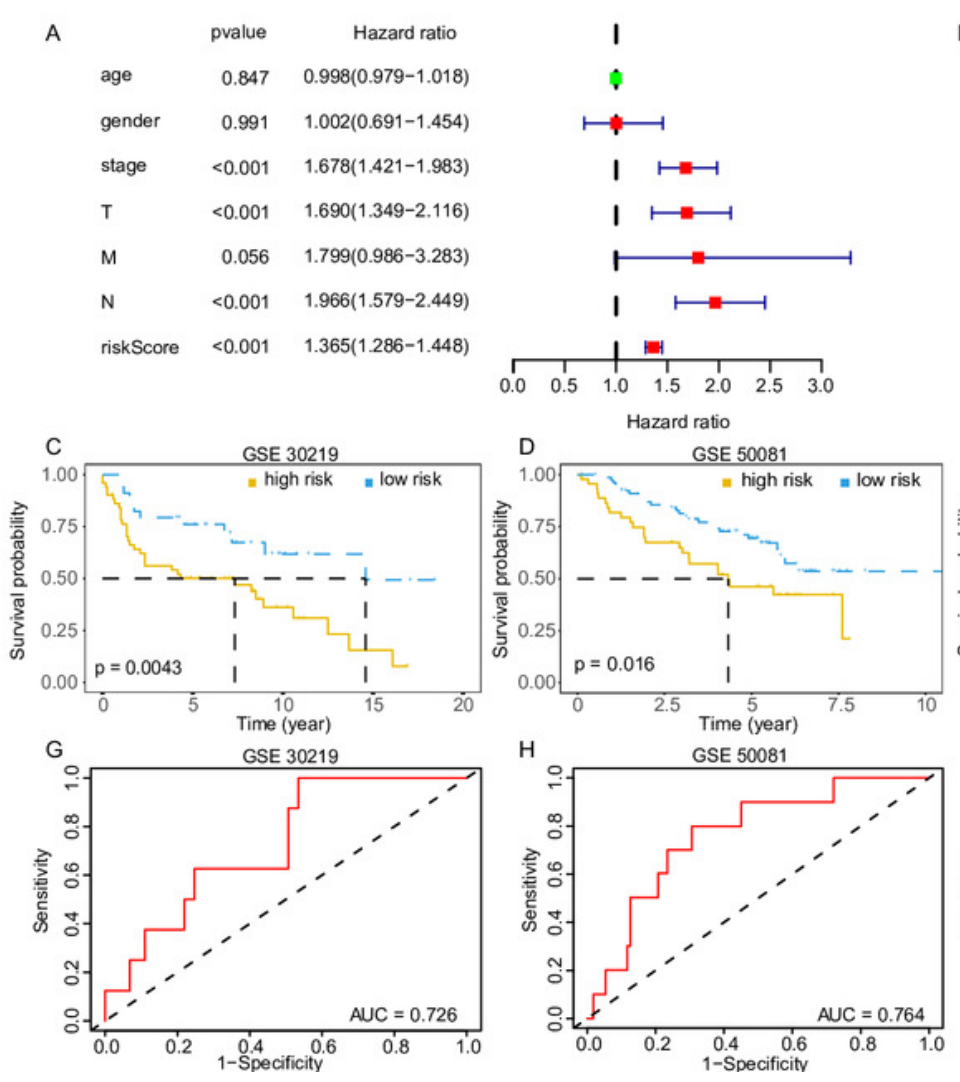
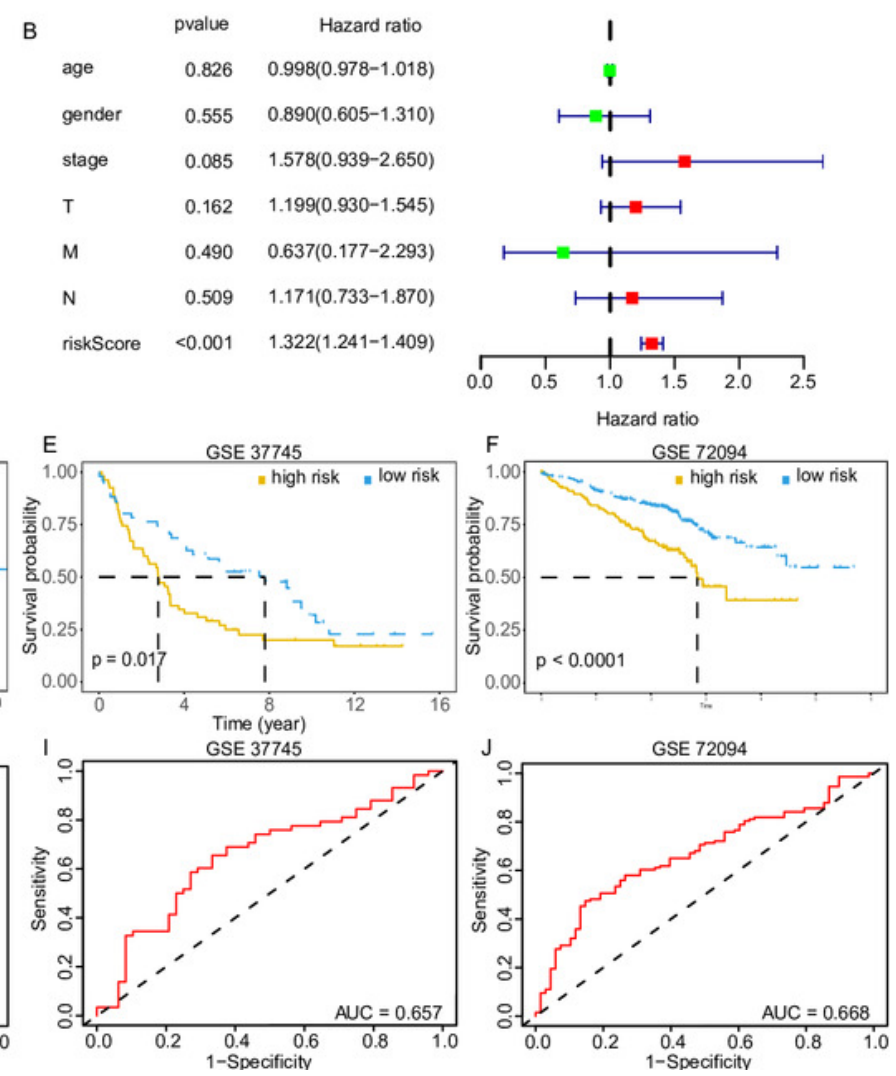
Figure 7

Prognostic nomogram and calibration plots of EGRS.

(A): The nomogram of EGRS. (B, C and D): calibration plots of nomogram predicting 1-year, 3year and 5-year survival, respectively.
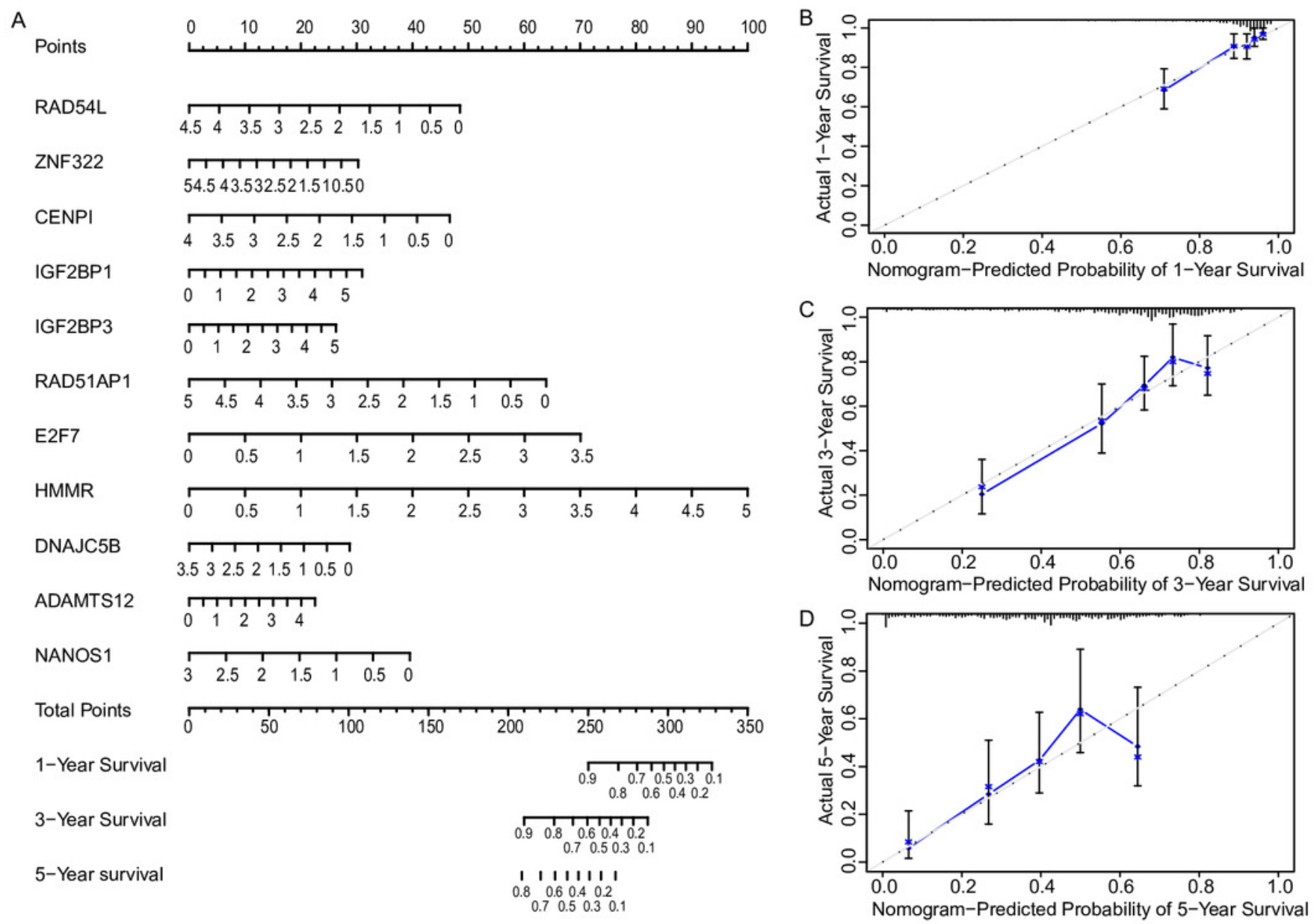